\title{
Endoscopic treatment of Zenker's diverticulum by LigaSure scalpel
}

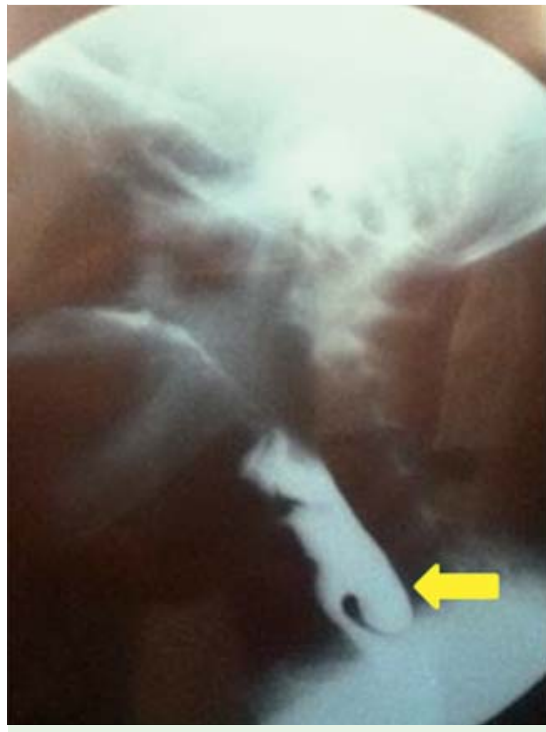

Fig. 1 Lateral view from a single-contrast esophagogram showing a barium-filled Zenker's diverticulum (arrow) posterior to the cervical esophagus.

Treatment of Zenker's diverticulum by flexible endoscopy is considered to be feasible, effective, and safe [1,2]. Endoscopic treatment consists of the division of the septum between the diverticulum and the esophagus, within which the cricopharyngeus muscle is contained $[1,2]$. This report describes the novel use of a LigaSure scalpel (Valleylab, Boulder, Colorado, USA) to perform flexible endoscopic diverticulectomy in a 3-cm Zenker's diverticulum in a 62-year-old woman with

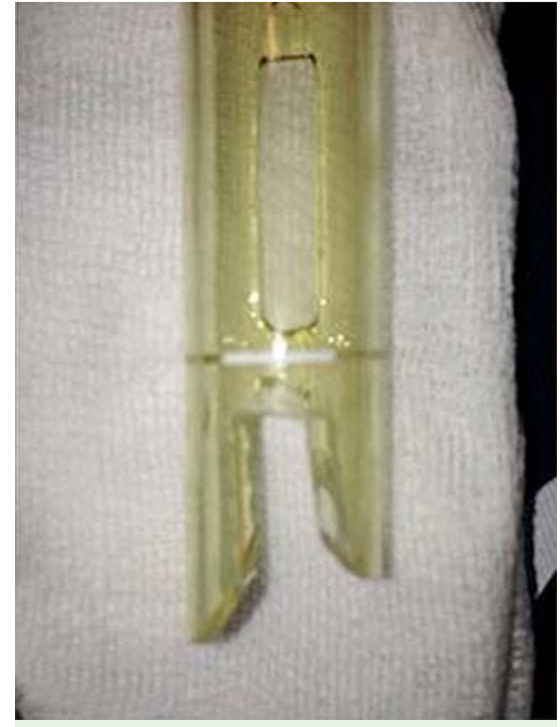

Fig. 2 A modified diverticuloscope with a double-lipped extremity that fits the anterior esophageal wall and the posterior wall of the diverticulum, leaving the septum in the center of the endoscopic field.

symptoms of dysphagia for both solids and liquids ( Fig. 1). We chose the LigaSure scalpel, which has been employed in laparoscopic surgery and provides adequate tissue dissection while achieving efficient and safe hemostasis, sealing vessels up to $7 \mathrm{~mm}$ in size [3]. This bipolar electrosurgical device delivers high-current, low-voltage electrical energy along with pressure that is exerted from the jaws of the device onto the tissue. The system monitors the energy expended while denaturing the collagen and elastin within the vessel walls [3].

A transparent overtube usually used for injection sclerotherapy of esophageal varices (Kawano overtube, $16 \mathrm{~mm}$ diameter) was modified and used as a "duckbeak diverticuloscope." The overtube allowed for excellent exposure of the septum and protection of the anterior esophageal and posterior diverticular walls, allowing the passage of the LigaSure scalpel ( $\bullet$ Fig. 2 ).

A standard videogastroscope (GIF-CV-160, $9.2 \mathrm{~mm}$; Olympus, Tokyo, Japan) and a stiff guidewire (Savary-Gilliard; Wilson Cook, Winston Salem, North Carolina, USA) were used to insert the diverticuloscope and achieve an adequate exposure of the septum of the Zenker's diverticulum. The LigaSure was then introduced through the diverticuloscope with endoscopic assistance ( Fig. 3 a). The septum of the Zenker's diverticulum was then safely cut up to the fundus of the diverticular sac without bleeding or perforation ( $\bullet$ Fig.3b). The procedure was completed by placing endoclips (Resolution Clip; Boston Scientific, Massachusetts, USA) at the base of the dissection ( Fig.3c). The patient made good progress, being able to tolerate liquids and pureed diet satisfactorily within 1 week, and progressing to a full diet the following week with no symptoms of dysphagia ( $\bullet$ Fig. 4 ).

Diathermic monopolar current, argon plasma coagulation, laser, and coagulation with an ultrasonically activated scalpel (Harmonic scalpel; Ethicon EndoSurgery) have all been used for the endoscopic treatment of Zenker's diverticulum with satisfactory results $[1,2,4]$. This case shows that endoscopic treatment of Zenker's diverticulum using a LigaSure scalpel
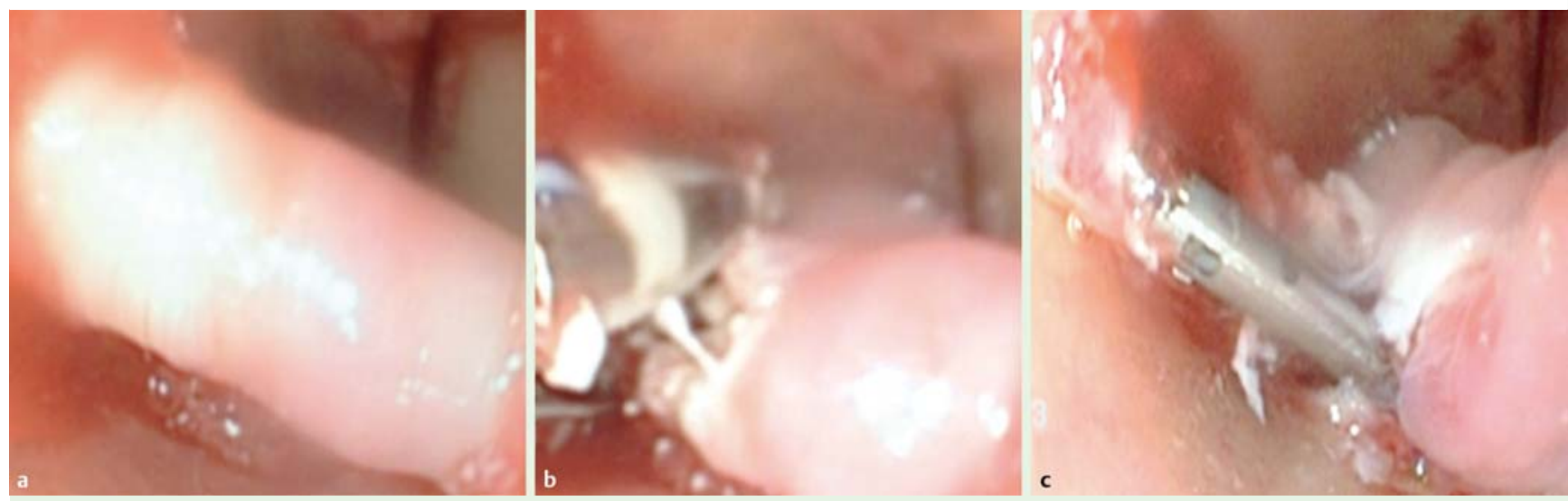

Fig. 3 Endoscopic views of the steps involved in treatment showing: a the cricopharyngeus muscle with a diverticuloscope in place, with the esophageal lumen above and the lumen of the diverticulum below; $\mathbf{b}$ the appearance after diverticulotomy with the LigaSure scalpel; $\mathbf{c}$ endoclips placed at the bottom of the diverticulum. 


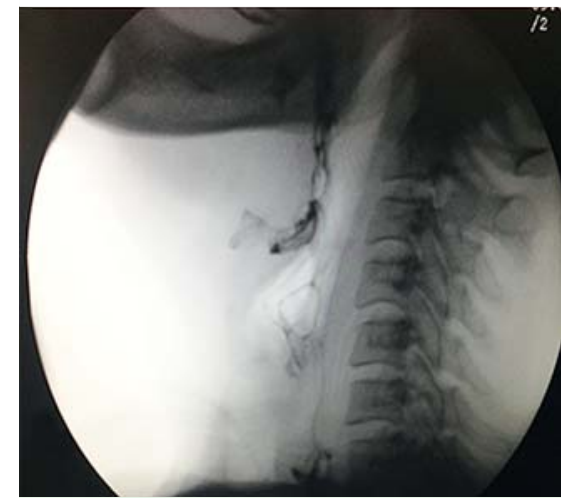

Fig. 4 Barium swallow after diverticulotomy showing no evidence of a contrast level within the diverticulum.

is a valid, easy, safe, and efficient alternative for treatment of a Zenker's diverticulum. Therefore, this technique should additionally be considered for endoluminal, minimally invasive treatment of Zenker's diverticulum.

Endoscopy_UCTN_Code_TTT_1AO_2AG

Competing interests: None

\section{Nicolás González ${ }^{1}$, Marcelo Viola ${ }^{2}$, Ximena Costa ${ }^{1}$, Alejandra Gamba ${ }^{1}$}

${ }^{1}$ Department of Gastroenterology "Prof. Henry Cohen," Faculty of Medicine, Hospital de Clínicas, Montevideo,

Uruguay

${ }^{2}$ Department of Surgery "Prof. Cesar Cannesa," Faculty of Medicine, Hospital de Clínicas, Montevideo, Uruguay

\section{References}

1 Huberty V, El Bacha S, Blero D et al. Endoscopic treatment for Zenker's diverticulum: long-term results (with video). Gastrointest Endosc 2013; 77: 701 - 707

2 Sakai P, Ishioka S, Maluf-Filho F et al. Endoscopic treatment of Zenker's diverticulum with an oblique-end hood attached to the endoscope. Gastrointest Endosc 2001; 54: $760-763$

3 Carbonell AM, Joels CS, Kercher KW et al. A comparison of laparoscopic bipolar vessel sealing devices in the hemostasis of small, medium, and large-sized arteries. J Laparoendosc Adv Surg Tech A 2003; 13: 377-380

4 Fama AF, Moore EJ, Kasperbauer JL. Harmonic scalpel in the treatment of Zenker's diverticulum. Laryngoscope 2009; 119: $1265-1269$
Bibliography

Dol http://dx.doi.org/

10.1055/s-0034-1365380

Endoscopy 2014; 46: E229-E230

(c) Georg Thieme Verlag KG

Stuttgart · New York

ISSN 0013-726X

\section{Corresponding author}

\section{Nicolás González, MD}

Department of Gastroenterology Hospital de Clínicas

Av Italia 2870-Piso 4

Montevideo 11600

Uruguay

Fax: +598-2-4808472

nicolasendoscopia@yahoo.es 